\title{
ON BIRNBAUM'S MEASURE OF COMPONENT IMPORTANCE OF COHERENT SYSTEMS
}

\author{
Yunus BULUT, Ahmet DEMIRALP, M. Şamil ŞIK *
}

İnönü Üniversitesi*, İnönü Üniversitesi**, İnönü Üniversitesi***

E-mail: ybulut79@gmail.com*;ahmt.dmrlp@gmail.com**

Copyright (C) 2015 Yunus BULUT, Ahmet DEMIRALP, M. Şamil ŞIK. This is an open access article distributed under the Eurasian Academy of Sciences License, which permits unrestricted use, distribution, and reproduction in any medium, provided the original work is properly cited.

\begin{abstract}
Together with rapidly developing technology, nowadays, plenty of complex systems have emerged and some algorithms for reliabilities of these systems have been developed. System analysts have realized that one of the factors which affects the system reliability is importance of the components forming the system, so they have developed various methods which measure component's importance. In this area, the first study was made by Birnbaum (Birnbaum, 1969:581-592). In this study, the reliabilities of coherent consecutive k-out-of-n:G systems and the Birnbaum's component importance of these systems are investigated. For this purpose, Birnbaum's importance measures of components of k-out-of-n:G systems are computed with Monte-Carlo simulations repeated by using different random numbers. In particular, the reliabilities of consecutive k-out-of-5:G systems and Birnbaum's importance measures of components which belong to these systems are analyzed.
\end{abstract}

Keywords: Coherent systems, System reliability, Consecutive k-out-of-n: G system, Birnbaum's measure of component importance, Monte-Carlo Simulation

\section{TUTARLI SISTEMLERIN BİRNBAUM BILLEŞEN ÖNEM ÖLÇÜMÜ ÜZERINNE}

Özet: Hızla gelişen teknoloji ile birlikte, günümüzde, pek çok karmaşık sisstem ortaya çıkmış ve bu sistemlerin güvenirlikleri için bazı algoritmalar geliştirilmiştir. Sistem analistleri, sistem güvenilirliğini etkileyen faktörlerden birinin sistemi oluşturan bileşenlerin önemi olduğunu fark etmiş ve böylece bileşenlerin önemini ölçen çeşitli metotlar geliştirmişlerdir. Bu alanda, ilk çalışma Birnbaum tarafından yapılmıştır (Birnbaum,1969:581-592). Bu çalışmada, tutarlı n'den ardıl k çıkışlı:G sistemlerin güvenilirlikleri ve bu sistemlerin Birnbaum bileşen önemi incelenmiştir. Bu amaçla, n'den ardıl k çıkışlı:G sistemlerin bileşenlerinin Birnbaum önem ölçümleri, farklı rassal sayılar kullanılarak tekrarlanan Monte-Carlo simülasyonları ile hesaplanmıştır. Özel olarak, 5'den ardıl k çıkışlı:G sistemlerin güvenilirlikleri ve bu sistemlere ait bileşenlerin Birnbaum önem ölçümleri analiz edilmiştir. 
Anahtar Kelimeler: Tutarlı sistemler, Sistem güvenilirliği, n'den ardıl k çıkışlı: G sistem, Birnbaum bileşen önem ölçümü, Monte-Carlo Simülasyonu

\section{Giriș}

En genel haliyle güvenilirlik, belirli bir yapıda düzenlenmiş bir veya daha fazla bileşenden oluşan bir sistemin yaşam olasılığı olarak tanımlanır. Literatürde ve günlük hayatta en çok kullanılan sistemler çeşitleri tutarlı sistemlerdir. Bir sistemin yapı fonksiyonu $\phi(x)$; her bağımsız $x_{i}(i \in[n]=\{1,2, \ldots, n\})$ bileşeni için monoton azalmayan bir fonksiyon ise ve sistem ilişkili bileşenlerden oluşuyorsa bu sisteme tutarlı sistem denir. Burada; $n$ tane bileşenden oluşan bir sistemdeki $i$. bileşenin durumu, $i \in[n]$ olmak üzere; $i$. bileşen $t$ anında çalışıyorsa $x_{i}=1$ ve $i$. bileşen $t$ anında çalışmıyorsa $x_{i}=0$ ile tanımlanır. $i$. bileşenin $T_{i}$ yaşam süresinin herhangi bir $t$ anından büyük olma olasılığ bileşen güvenilirliğidir ve $i \in[n]$ için $x_{i}=1$ olma olasılığ $P\left(T_{i}>t\right)=P\left(x_{i}=1\right)=p_{i}$ ile gösterilir. Benzer şekilde, sistemin durumu

$$
\phi(\boldsymbol{x})=\left\{\begin{array}{l}
1, \quad \text { sistem } t \text { anında çalışıyorsa } \\
0, \quad \text { sistem } t \text { anında çalışmıyorsa }
\end{array}\right.
$$

dönüşümü ile tanımlanır. $\phi(x)$ fonksiyonu, $\boldsymbol{x}=\left(x_{1}, x_{2}, \ldots, x_{n}\right) \in\{0,1\}^{n}$ olmak üzere, $n$ bileşenden oluşan bir sistemin yapı fonksiyonudur (Barlow vd., 1975:1-20; Rausand vd., 2004:118-133; Kuo vd., 2003:85-95).

Sistemin $t$ anında çalışıyor olma olasılığına, yani $\phi(x)=1$ olma olasılığına sistem güvenilirliği denir. Sistemin güvenilirliği, $\boldsymbol{p}=\left(p_{1}, p_{2}, \ldots, p_{n}\right)$ olmak üzere, $\quad h(\boldsymbol{p})=$ $P(\phi(\boldsymbol{x})=1)=E \phi(\boldsymbol{x})$ eşitliği ile hesaplanır (Barlow vd., 1975:20-52; Rausand vd., 2004:160-161). Bazen yap1 fonksiyonu yardımıyla sistemin güvenilirliğini incelemek zor olduğundan, yapı fonksiyonunun duali ile işlem yapılır. Bir $\phi(x)$ yapı fonksiyonunun duali, $1-\boldsymbol{x}=\left(1-x_{1}, 1-x_{2}, \ldots, 1-x_{n}\right) \quad$ olmak üzere, $\phi^{D}(\boldsymbol{x})=1-\phi(1-\boldsymbol{x})$ eşitliği ile tanımlanır (Kuo vd., 2003:85-95; Barlow vd.,1975:12).

Teknolojik gelişmeler, herhangi bir tutarlı sistemin güvenilirliği ve dizaynı için sistemi oluşturan bileșenlerin güvenilirliklerinin önemini arttırmıştır. $\mathrm{Bu}$ yüzden, literatürde bir bileşenin, sistem için ne kadar önemli olduğunu hesaplayan Birnbaum önemi (Birnbaum, 1969: 581-592), Barlow-Proschan önemi (Barlow vd., 1975: 153-173), Fussel-Vesely önemi (Fussel, 1975: 169-174), Natvig önemi (Natvig, 1979: 319-330), Yapı önemi (Wu, 2005: 6375) gibi çeşitli önem ölçümleri geliştirilmiştir. Öte yandan, Birnbaum önem ölçümü yardımıyla maliyet tabanlı bir önem ölçümü geliştirilmiştir (Wua vd., 2013: 189-195). Ayrıca, Birnbaum önem ölçümü, tutarlı olmayan sistemler için incelenmiştir (Andrews vd., 2003). Bayesian ağ temelli ikili tutarlı sistemlerde farklı bileşenler için Birnbaum önem ölçümünün değişen özellikleri ve ilişkileri incelenmiştir (Zhiqiang vd., 2013: 203-219). Sistem bileşenlerinin Birnbaum ve Fussel-Vesely önem ölçümleri yardımıyla kritik bağ ile bileşen önem ölçümleri arasında çeşitli ilişkiler türetilmiştir (Meng, 2000: 55-60). Uygulama alanında 
ise rüzgâr türbininin ana sistemi ve onun alt sistemleri için güvenilirlik önem analizi yapılmıştır (Selwyn, 2011).

$\mathrm{Bu}$ çalışmada, $1 \leq k \leq 5$ olmak üzere ardıl koo5:G tutarlı sistemlerin bileşenleri için Birnbaum bileşen önem ölçümü, Monte-Carlo simülasyonu yardımıyla çeşitli gözlem değerleri için incelenmiştir.

\section{2. n'den $\boldsymbol{k}$ Çıkışıı Sistemler}

$n$ bileşenden en az $k$ tanesi çalıştığında $n$-bileşenli sistem çalışıyorsa bu sisteme n'den $k$ çıkışlı: $G$ sistem denir. Eğer $n$ bileşenden en az $k$ tanesi hatalı iken $n$-bileşenli sistem de hatalıysa bu sisteme $n$ 'den $k$ çıkışlı: $F$ sistem adı verilir. Bu tanımlardan bir $n$ 'den $k$ çıkışlı: $G$ sistemin, $n$ 'den $(n-k+1)$ çıkışlı: $F$ sisteme denk olduğu söylenebillir. $n$ 'den $k$ çıkışlı sistem, sıklıkla, ya $G$ sistemi ya $F$ sistemi ya da her ikisini de göstermek için kullanılır. Hem paralel hem de seri yapılar $n$ 'den $k$ çıkışlı sistemlerin özel durumlarıdır. Bir seri sistem $n$ 'den 1 çıkışlı: $F$ sisteme ve $n$ 'den $n$ çıkışlı: $G$ sisteme denktir. Benzer şekilde bir paralel sistem de $n$ 'den $n$ çıkışlı: $F$ sisteme ve $n$ 'den 1 çıkışlı: $G$ sisteme denktir (Kuo vd., 2003:231-255).

n'den ardıl $k$ çılkş̧lı: G sistemlerle ilgili ilk çalıșmayı Tong vermiştir (Tong,1985:386393 ) ve $n$ den ardıl $k$ çıkışlı: $G$ sistem konsepti en iyi Kuo vd. tarafindan açıklanmıştır (Kuo vd.,1990:325-363). Bu yazarlara göre; $n$ 'den ardıl $k$ çıkışlı: $G$ ve $F$ sistemler birbirlerinin aynadaki yansımaları gibidir. Gerçekten de $n$ 'den ardıl $k$ çıkışıı: $F$ ve $G$ sistemlerin birbirlerinin dualleri olduğu kolayca gösterilebilir. Duallikten, $n$ 'den $\operatorname{ard} 1 l \quad k$ çıkışlı: $F$ sistemdeki bir minimal kesen kümesinin, $n$ 'den ardıl $k$ çıkışlı: $G$ sistemin bir minimal yol kümesi olduğu görülür.

Örnek 1: $n$ 'den $\operatorname{ard} 11 k$ çıkışlı: $G$ sistemlerin özel hali olarak 5'den ardıl 1 çıkışlı: $G$ sistem için minimal başarı yol kümeleri kümesi $\{\{1\},\{2\},\{3\},\{4\},\{5\}\}$ olduğundan sistemin yap1 fonksiyonu,

$$
\phi(\boldsymbol{x})=1-\left(1-x_{1}\right)\left(1-x_{2}\right)\left(1-x_{3}\right)\left(1-x_{4}\right)\left(1-x_{5}\right)
$$

ve (2.1)'in beklenen değeri alınırsa sistem güvenilirliği,

$$
h(\boldsymbol{p})=1-\left(1-p_{1}\right)\left(1-p_{2}\right)\left(1-p_{3}\right)\left(1-p_{4}\right)\left(1-p_{5}\right)
$$

şeklinde elde edilir.

\section{Yapısal ve Birnbaum Bileşen Önem Ölçümü}

Bir sistemdeki bazı bileşenler, sistem güvenilirliği için diğer bileşenlerden daha önemli olabilir. Örneğin, bir sistemin diğer bileşenleri ile seri bağlanmış bir bileşen, sistemin diğer 
bileşenlerinden daha önemlidir veya hiç olmazsa onlarla aynı öneme sahiptir. Bu durum mühendisler ve sistem analistleri için sistemin tasarımında önem arz etmektedir. Çünkü bileşenlerin iyileştirilmesi işlemlerine kıyasla, bileşenlerin güvenilirliklerindeki küçük değişimler karşısında sistem güvenilirliğinin hassasiyetini ölçmek daha önceliklidir (Aven vd., 2013: 29). Genellikle bileşen önem ölçümleri, bileşenlerin sistemdeki arızalara etkisini göz önüne alarak bileşenleri önem seviyesine göre bir sıraya dizer (Wang vd., 2004). Bir bileşenin önemi; bileşenin sistemdeki yeri, bileşenin güvenilirliği ve de bileşenin güvenilirliğinde yaptığımız tahmindeki belirsizlik faktörlerine bağlıdır (Rausand vd., 2004: 183-207).

Tanım 1. Birnbaum bileşen önem ölçümü, bir bileşenin sistem güvenilirliği üzerindeki etkisini temsil eder (Birnbaum, 1969: 581-592). $i$. bileşenin Birnbaum ölçümü, sistem güvenilirliğinin, $p_{i}(t)$ 'ye göre kısmi türevi alınarak bulunur. Yani, $i$. bileşenin $t$ anındaki önem ölçümü,

$$
I_{i}^{B}(t)=\frac{\partial h(\boldsymbol{p}(t))}{\partial p_{i}(t)}, \quad i \in[n]
$$

eşitliğgi ile tanımlanır. Buradan; $I_{i}^{B}(t)$ ölçümü büyüdükçe, $t$ anında $i$. bileşenin güvenilirliğindeki küçük bir değişimin, sistem güvenilirliğinde büyük bir değişiklik meydana getireceği söylenebilir. (3.1) eşitliğinde $p_{i}=0.5$ alınırsa yapısal önem ölçümü elde edilir (Rausand vd., 2004:183-207). $i \in[n]$ için sistemin $t$ anında arızalanma fonksiyonu $Q_{0}(t)=$ $1-h(\boldsymbol{p}(t))$ ve $i$. bileşenin $t$ anında çalışmama olasılı̆̆ 1 $q_{i}(t)=1-p_{i}(t)$ olarak alınırsa Birnbaum ölçümü;

$$
I_{i}^{B}(t)=\frac{\partial Q_{0}(t)}{\partial q_{i}(t)}
$$

eşitliği ile de ifade edilebilir. $i$. bileşenin sistemin güvenilirliğine etkisini hesaplamanın zor olduğu durumlarda, sistem $i$. bileşeninin durumuna göre;

$$
\begin{aligned}
\operatorname{Pr}(\phi(\boldsymbol{x}) \text { çalışır }) \quad & =\operatorname{Pr}(\text { i.bileşen çalışıyor }) \operatorname{Pr}(\phi(\boldsymbol{x}) \text { çalışıyor } \mid \text { i. bileşen çalıșıyor }) \\
& +\operatorname{Pr}(\text { i.bileşen arızalı }) \operatorname{Pr}(\phi(\boldsymbol{x}) \text { çalışıyor } \mid \text { i. bileşen arızalı })
\end{aligned}
$$

mantığ 1 ile ayrıştırılabilir. Bu ayrışıma esas ayrışım denir ve matematiksel olarak; $i \in[n]$ için, $\phi(\boldsymbol{x})=x_{i} \phi\left(1_{i}, \boldsymbol{x}\right)+\left(1-x_{i}\right) \phi\left(0_{i}, \boldsymbol{x}\right)$ şeklinde ifade edilir. Burada; $a=0,1$ olmak üzere $(a, \boldsymbol{x})=\left(x_{1}, x_{2}, \ldots, x_{i-1}, a, x_{i+1}, \ldots x_{n}\right)$ dir (Kuo vd., 2003:140-145; Rausand vd., 2004:136).

Bağımsız $n$-bileşen için sistem güvenilirliği, esas ayrışım kullanılarak, aşağıdaki gibi $p_{i}(t)$ bileşen güvenilirliğinin doğrusal bir fonksiyonu olarak yazılabilir.

$$
\begin{aligned}
h(\boldsymbol{p}(t)) & =p_{i}(t) h\left(1_{i}, \boldsymbol{p}(t)\right)+q_{i}(t) h\left(0_{i}, \boldsymbol{p}(t)\right) \\
& =p_{i}(t)\left[h\left(1_{i}, \boldsymbol{p}(t)\right)-h\left(0_{i}, \boldsymbol{p}(t)\right)\right]+h\left(0_{i}, \boldsymbol{p}(t)\right), \quad i \in[n]
\end{aligned}
$$


Burada, $h\left(1_{i}, \boldsymbol{p}(t)\right), t$ anında $i$. bileşenin çalıştığ biliniyorken sistemin de çalışıyor olma olasılığını ve $h\left(0_{i}, \boldsymbol{p}(t)\right), t$ anında $i$. bileşenin çalışmadığı biliniyorken sistemin çalışıyor olma olasılığını göstermektedir. (3.3) ifadesinin $p_{i}(t)$ 'ye göre kısmi türevi alınırsa,

$$
I_{i}^{B}(t)=h\left(1_{i}, \boldsymbol{p}(t)\right)-h\left(0_{i}, \boldsymbol{p}(t)\right)
$$

ve sistem güvenilirliği tanımdan

$$
\begin{aligned}
I_{i}^{B}(t) & =E\left[\phi\left(1_{i}, \boldsymbol{X}(t)\right)\right]-E\left[\phi\left(0_{i}, \boldsymbol{X}(t)\right)\right] \\
& =E\left[\phi\left(1_{i}, \boldsymbol{X}(t)\right)-\phi\left(0_{i}, \boldsymbol{X}(t)\right)\right] \\
& =\operatorname{Pr}\left[\phi\left(1_{i}, \boldsymbol{X}(t)\right)-\phi\left(0_{i}, \boldsymbol{X}(t)\right)=1\right]
\end{aligned}
$$

eşitlikleri kolayca elde edilebilir (Kuo vd., 2003:193-194)

$i$. bileşenin Birnbaum önem ölçümü sadece sistemin yapısına ve $i$. bileşen hariç diğer bileşenlerin güvenilirliklerine bağlıdır. Yani $I_{i}^{B}(t)$, $i$. bileşenin $p_{i}(t)$ güvenilirliğinden bağımsızdır. Bu, Birnbaum ölçümünün bir zayıflığı olarak düşünülebilir (Rausand vd., 2004:185-186).

Örnek 2: 5'den ard11 1 çıkışlı sistemin 1. bileşeni için Birnbaum önem ölçümü, (2.2)'ye (3.1) uygulanarak,

$$
\begin{aligned}
I_{1}^{B}(t)= & 1-p_{2}-p_{3}-p_{4}-p_{5}+p_{2} p_{3}+p_{2} p_{4}+p_{2} p_{5}+p_{3} p_{4}+p_{3} p_{5}+p_{4} p_{5} \\
& -p_{2} p_{3} p_{4}-p_{3} p_{4} p_{5}-p_{2} p_{4} p_{5}-p_{2} p_{3} p_{5}+p_{2} p_{3} p_{4} p_{5}
\end{aligned}
$$

\begin{tabular}{|c|c|c|c|c|c|}
\hline & & & & & \\
\hline & $>\mathrm{I}^{\mathrm{B}}{ }_{4}>\mathrm{I}^{\mathrm{B}}{ }_{5}>$ & $\begin{array}{l}\mathrm{I}_{2}^{\mathrm{B}}>\mathrm{I}_{4}^{\mathrm{B}}>\mathrm{I}_{3}^{\mathrm{B}}>\mathrm{I}_{5}^{\mathrm{B}}> \\
\mathrm{I}_{1}^{\mathrm{B}}\end{array}$ & $\begin{array}{l}\mathrm{I}_{3}^{\mathrm{B}}>\mathrm{I}^{\mathrm{B}}{ }_{4}>\mathrm{I}^{\mathrm{B}}{ }_{2}>\mathrm{I}^{\mathrm{B}}{ }_{5}> \\
\mathrm{I}_{1}^{\mathrm{B}}\end{array}$ & $\begin{array}{l}\mathrm{I}_{4}^{\mathrm{B}}>\mathrm{I}_{2}^{\mathrm{B}}=\mathrm{I}_{3}^{\mathrm{B}}>\mathrm{I}_{5}^{\mathrm{B}}> \\
\mathrm{I}_{1}^{\mathrm{B}}\end{array}$ & $\begin{array}{l}\mathrm{I}_{1}^{\mathrm{B}}>\mathrm{I}^{\mathrm{B}}{ }_{2}>\mathrm{I}^{\mathrm{B}}{ }_{4}>\mathrm{I}^{\mathrm{B}}{ }_{3}= \\
\mathrm{I}^{\mathrm{B}}{ }_{5}\end{array}$ \\
\hline 3 & $\begin{array}{l}\mathrm{I}_{4}^{\mathrm{B}}>\mathrm{I}_{5}^{\mathrm{B}}>\mathrm{I}_{3}^{\mathrm{B}}>\mathrm{I}_{1}^{\mathrm{B}}> \\
\mathrm{I}_{2}^{\mathrm{B}}{ }_{2}\end{array}$ & $\begin{array}{l}\mathrm{I}_{4}^{\mathrm{B}}>\mathrm{I}_{2}{ }_{2}>\mathrm{I}_{3}^{\mathrm{B}}>\mathrm{I}_{5}^{\mathrm{B}}> \\
\mathrm{I}^{\mathrm{B}}{ }_{1}\end{array}$ & $\begin{array}{l}\mathrm{I}_{3}^{\mathrm{B}}>\mathrm{I}^{\mathrm{B}}{ }_{4}>\mathrm{I}_{2}{ }_{2}>\mathrm{I}_{5}^{\mathrm{B}}> \\
\mathrm{I}^{\mathrm{B}}{ }_{1}\end{array}$ & $\begin{array}{l}\mathrm{I}_{2}^{\mathrm{B}}>\mathrm{I}_{3}^{\mathrm{B}}>\mathrm{I}_{4}^{\mathrm{B}}>\mathrm{I}_{5}^{\mathrm{B}}> \\
\mathrm{I}_{1}^{\mathrm{B}}\end{array}$ & $\begin{array}{l}\mathrm{I}_{1}^{\mathrm{B}}>\mathrm{I}_{2}^{\mathrm{B}}>\mathrm{I}_{3}^{\mathrm{B}}>\mathrm{I}_{5}^{\mathrm{B}}> \\
\mathrm{I}^{\mathrm{B}}{ }_{4}\end{array}$ \\
\hline$C$ & $\begin{array}{l}\mathrm{I}_{5}^{\mathrm{B}}>\mathrm{I}_{3}^{\mathrm{B}}>\mathrm{I}_{2}^{\mathrm{B}}>\mathrm{I}_{4}^{\mathrm{B}}> \\
\mathrm{I}_{1}^{\mathrm{B}}\end{array}$ & $\begin{array}{l}\mathrm{I}_{4}^{\mathrm{B}}>\mathrm{I}_{2}{ }_{2}>\mathrm{I}_{3}^{\mathrm{B}}>\mathrm{I}^{\mathrm{B}}= \\
\mathrm{I}^{\mathrm{B}}{ }_{1}\end{array}$ & ${ }_{4}>\mathrm{I}^{\mathrm{B}}{ }_{2}>\mathrm{I}^{\mathrm{B}}{ }_{5}>$ & $\begin{array}{l}\mathrm{I}_{2}^{\mathrm{B}}>\mathrm{I}_{4}^{\mathrm{B}}>\mathrm{I}_{3}^{\mathrm{B}}>\mathrm{I}_{5}^{\mathrm{B}}> \\
\mathrm{I}_{1}^{\mathrm{B}}\end{array}$ & $\begin{array}{l}\mathrm{I}_{1}^{\mathrm{B}}>\mathrm{I}^{\mathrm{B}}{ }_{2}=\mathrm{I}_{5}^{\mathrm{B}}>\mathrm{I}^{\mathrm{B}}{ }_{3}> \\
\mathrm{I}^{\mathrm{B}}{ }_{4}\end{array}$ \\
\hline$L$ & $\begin{array}{l}\mathrm{I}_{1}^{\mathrm{B}}>\mathrm{I}_{5} \mathrm{~B}^{\mathrm{B}} \mathrm{I}_{3}^{\mathrm{B}}>\mathrm{I}_{4}^{\mathrm{B}}> \\
\mathrm{I}_{2}^{\mathrm{B}}\end{array}$ & $\begin{array}{l}\mathrm{I}_{4}^{\mathrm{B}}>\mathrm{I}_{2}{ }_{2}>\mathrm{I}_{3}^{\mathrm{B}}>\mathrm{I}_{5}^{\mathrm{B}}> \\
\mathrm{I}^{\mathrm{B}}{ }_{1}\end{array}$ & $\begin{array}{l}\mathrm{I}_{3}^{\mathrm{B}}>\mathrm{I}^{\mathrm{B}}{ }_{2}=\mathrm{I}^{\mathrm{B}}{ }_{4}>\mathrm{I}^{\mathrm{B}}{ }_{5} \mathrm{P} \\
\mathrm{I}^{\mathrm{B}}{ }_{1}\end{array}$ & $\begin{array}{l}\mathrm{I}_{4}^{\mathrm{B}}>\mathrm{I}_{2}{ }_{2}>\mathrm{I}_{3}^{\mathrm{B}}>\mathrm{I}^{\mathrm{B}}= \\
\mathrm{I}^{\mathrm{B}}{ }_{1}\end{array}$ & $\begin{array}{l}\mathrm{I}_{4}^{\mathrm{B}}>\mathrm{I}^{\mathrm{B}}{ }_{2}>\mathrm{I}^{\mathrm{B}}{ }_{3}>\mathrm{I}^{\mathrm{B}}{ }_{5} \mathrm{P} \\
\mathrm{I}^{\mathrm{B}}{ }_{1}\end{array}$ \\
\hline & $\begin{array}{l}\mathrm{I}_{3}^{\mathrm{B}}=\mathrm{I}_{5}^{\mathrm{B}}>\mathrm{I}_{2}{ }_{2}=\mathrm{I}_{4}^{\mathrm{B}}> \\
\mathrm{I}^{\mathrm{B}}{ }_{1}\end{array}$ & $\begin{array}{l}\mathrm{I}_{4}^{\mathrm{B}}>\mathrm{I} \\
\mathrm{I}^{\mathrm{B}}{ }_{1}\end{array}$ & $\begin{array}{l}\mathrm{I}_{3}^{\mathrm{B}}>\mathrm{I}^{\mathrm{B}}{ }_{4}>\mathrm{I}^{\mathrm{B}}>{ }_{2}>\mathrm{I}_{5}^{\mathrm{B}}> \\
\mathrm{I}^{\mathrm{B}}{ }_{1}\end{array}$ & $\begin{array}{l}\mathrm{I}_{2}{ }_{2}>\mathrm{I}_{3}{ }_{3}=\mathrm{I}_{4}^{\mathrm{B}}>\mathrm{I}^{\mathrm{B}}> \\
\mathrm{I}^{\mathrm{B}}{ }_{5}\end{array}$ & $\begin{array}{l}\mathrm{I}_{2}^{\mathrm{B}}>\mathrm{I}^{\mathrm{B}}{ }_{17} \\
\mathrm{I}_{5}^{\mathrm{B}}\end{array}$ \\
\hline
\end{tabular}

olarak elde edilir. Benzer şekilde, 5'den ardıl $k$ çıkışlı: $G$ sistemlerin tüm bileşenlerinin Birnbaum önem ölçümleri elde edilebilir. Bu hesaplamalar yardımıyla, 5'den ardıl $k$ çıkışlı: $G$ sistemler için 5 ayrı Monte-Carlo simülasyonu yapılmış ve sonuçlar aşağıdaki tabloda verilmiştir.

Tablo 1: Ard11 koo5: $G$ sistemler için bileşenlerin önem ölçümleri 
$\mathrm{Bu}$ tabloya göre, örneğin $\mathrm{A}$ denemesinde 5 'den ardıl 3 çıkışlı: $G$ sistem için $\mathrm{I}^{\mathrm{B}}{ }_{3}>\mathrm{I}^{\mathrm{B}}{ }_{4}>\mathrm{I}^{\mathrm{B}}{ }_{2}>\mathrm{I}^{\mathrm{B}}{ }_{5}>\mathrm{I}^{\mathrm{B}}{ }_{1}$ eşitsizliği elde edilmiştir. Buna göre 3. bileşen en önemli ve 1. bileşen diğerlerine göre en önemsiz bileşen olarak tespit edilmiştir. Yani; 3. bileşenin sistemden çıkarılması 1. bileşenin sistemden çıkarılmasından daha tehlikelidir.

Örnek 3. 5'den ardıl 3 çıkışlı:G sistemin bileşenlerinin Birnbaum önem ölçümleri için Tablo 1'deki denemelerden farklı olarak üç farklı simülasyon yapılmış ve simülasyonların yüzdelik karşılıkları Grafik 1'de verilmiştir. Bu grafikten de görüldügüü üzere üç simülasyon sonucunda da 3. bileşen sistem için en önemli bileşendir.

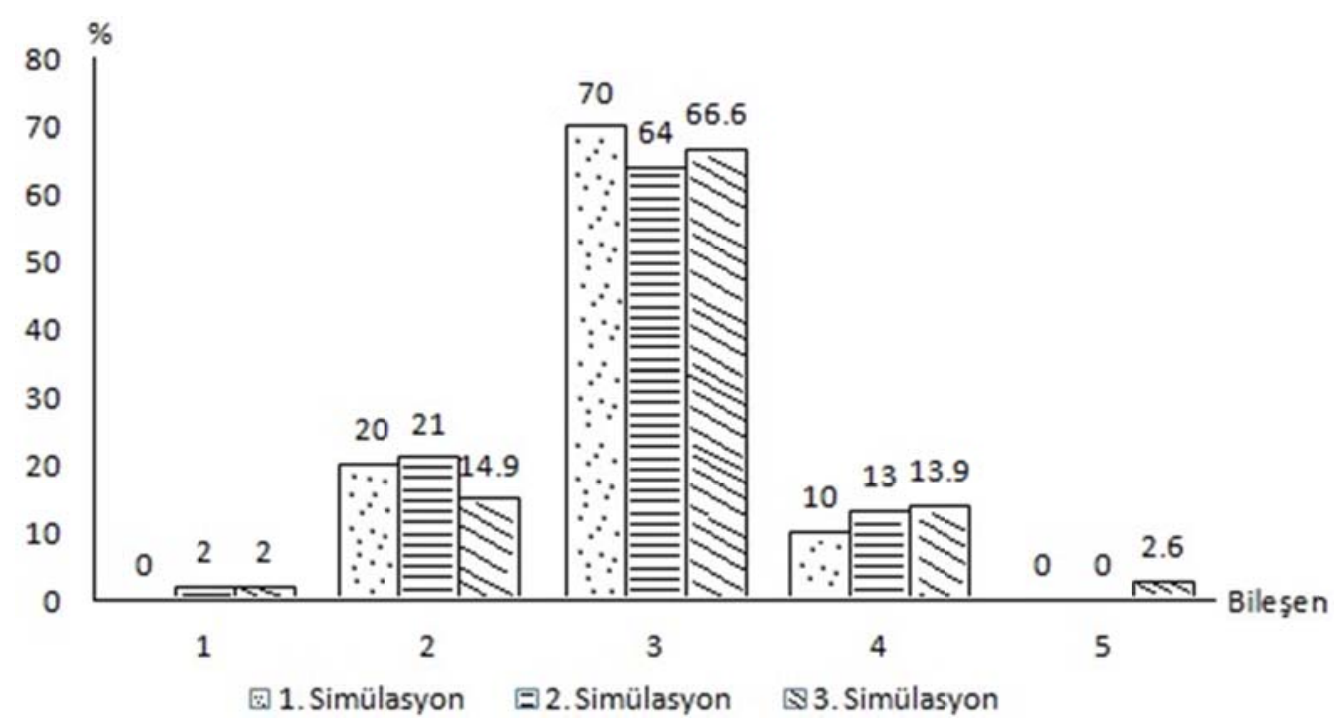

Grafik 1: Ard11 3oo5: $G$ sistem için bileşenlerin önem ölçüm dağılımı

Ayrıca, $p_{i}=0.5$ için bileşenlerin Birnbaum yapısal önem ölçümleri arasındaki ilişki $B_{\phi}(3)>B_{\phi}(2)=B_{\phi}(4)>B_{\phi}(1)=B_{\phi}(5)$ şeklinde elde edilmiştir. Bu sonucun yapılan Monte-Carlo simülasyonu ile uyum içinde olduğu görülmektedir.

Örnek 4. 5'den ardıl 1 çıkışlı:G sistem ve bu sistemin duali olan 5'den ardıl 5 çıkışlı:G sistem için bileşenlerin aynı güvenilirlik $\left(p=p_{1}=p_{2}=p_{3}=p_{4}=p_{5}\right)$ değerini aldı ̆̆ kabul edilirse; bileşenlerin Birnbaum bileşen önem ölçümleri eşit olur. $\mathrm{Bu}$ sistemlerin güvenilirlikleri ile bileşenlerin Birnbaum önem ölçümleri arasındaki ilişki aşağıda, sırasıyla, Grafik 2 ve Grafik 3'de gösterilmiştir. 


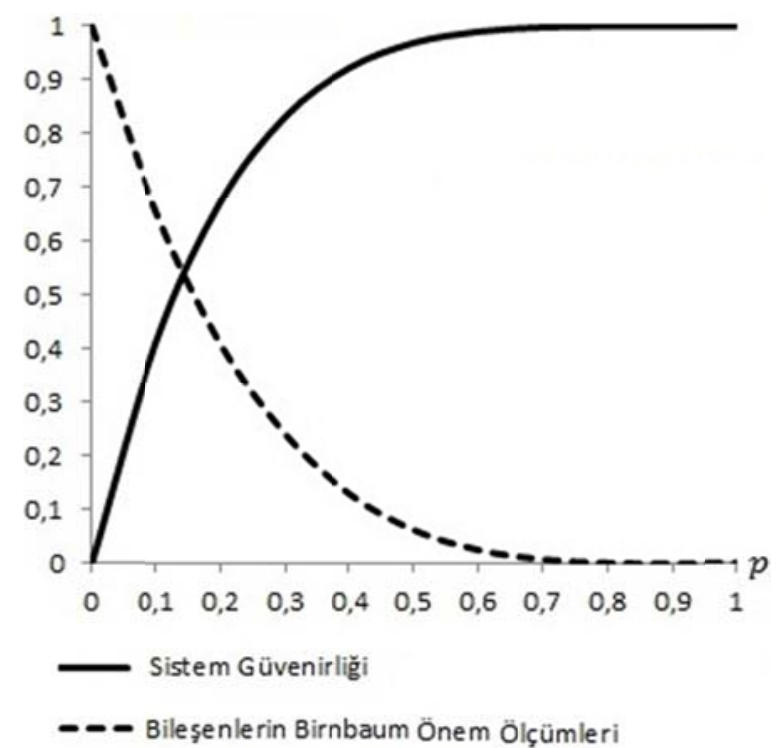

Grafik 2: Ardıl 10o5:G

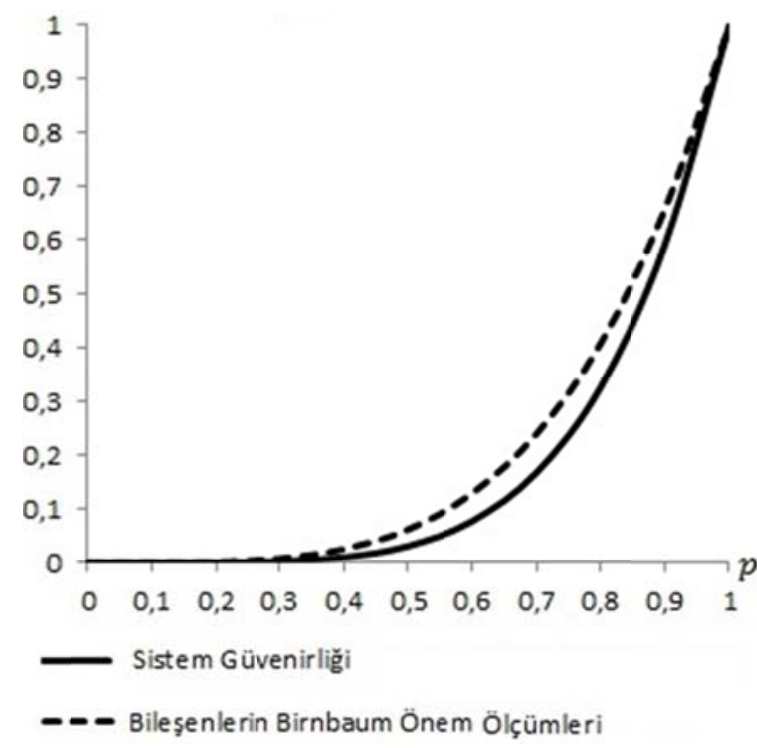

Grafik 3: Ardıl 5oo5:G

\section{Sonuçlar}

(3.1) eşitliğinde de görüldüğü gibi $i$. bileşenin Birnbaum önem ölçümü, sadece sistemin yapısına ve $i$. bileşen hariç diğer bileşenlerin güvenilirliklerine bağlıdır. Bu durum, Birnbaum ölçümünün bir zayıflığı olarak düşünülebilir. Bu dezavantajına rağmen diğer bazı önem ölçümleri de Birnbaum önem ölçümünden hesaplandığından uygulama alanı oldukça geniştir.

Tablo 1'deki beş denemenin dördünde, 5'den ardıl 2 çıkışlı:G sistemin 4 . bileşeninin Birnbaum önem ölçümü yüzdesi, diğer bileşenlerin yüzdesinden daha yükssek çıkmıştır. Fakat Tablo 1 rassal sayı üretilerek elde edildiğinden, bileşenlerin önem ölçümlerinin kıyaslanmasında genel bir yargıya varılamamaktadır. Yani, bileşenlerin Birnbaum önem ölçümü bir sistemin bileşenlerinin farklı güvenilirlik değerleri için farklı değerler alabilir. Öte yandan, Grafik 2 ve Grafik 3'de görüldüğü gibi aynı bileşen önemine sahip bileşenlerden oluşan 5'den ardıl 1 çıkışlı:G sistemlerde bileşenlerin Birnbaum önem ölçümü arttıkça sistemin güvenilirliği azalmış, fakat 5'den ardıl 5 çıkışlı:G sistemlerde, bileşenlerin Birnbaum önem ölçümleri arttıkça sistemin güvenilirliği artmıştır.

Bir bileşenin önem ölçümü sistemin dizaynına ve bileşenlerin güvenilirliklerine bağlı olduğundan, sistemin dizaynı için bazı genellemeler yapılabilir. Örneğin; sisteme seri bağl1 olan bir bileşen diğer bileşenlerden daha önemli olabilir. 


\section{REFERENCES}

- Andrews John D. and Beeson Sally (2003), Birnbaum's Measure of Component Importance for Noncoherent Systems, IEEE TRANSACTIONS ON RELIABILITY, VOL. 52, NO. 2, JUNE.

- Aven T., Jensen U., (2013), Stochastic Models in Reliability, Springer Science \& Business Media

- Barlow, R.E., Proschan, F. (1975), Statistical Theory of Reliability and Life Testing: Probability Models, s.1-25, Holt, Rinehart and Winston, Inc. New York.

- Barlow, R.E., Proschan, F. (1975), Importance of system components and fault tree events, Stochastic Processes and their Application 3 (2),153-173.

- Birnbaum, Z. (1969), On the importance of different components in a multicomponent system, Multivariate Analysis, vol. II, Academic Press, New York, pp.581-592.

- Fussel, J. B. (1975), How to hand-calculate system reliability and safety characteristics, IEEE Transactions on Reliability R-24 (3), 169-174.

- Kuo, W., Zuo, M. (2003), Optimal Reliability Modeling: Principles and Applications, John Wiley\&Sons, Inc., Hoboken, New Jersey.

- Kuo W., Zhang W. and Zuo M. (1990), A consecutive -k-out-of-n:G system:The mirror image of a consecutive-k-out-of- $n: F$ system, IEEE Transactions on Reliability, R-39(2):244-253.

- Meng F. C. (2000), Relationships of Fussell-Vesely and Birnbaum importance to structural importance in coherent systems, Reliability Engineering, and System Safety 67, 55-60.

- Natvig, B.(1979) , A suggestion of a new measure of importance of system components, Stochastic Processes and their Applications 9 (3), 319-330.

- Rausand, M., Hoyland, A. (2004), System Reliability Theory: Models, Statistical Methods, and Applications, John Wiley\&Sons, Inc., Hoboken, New Jersey.

- Selwyn T. Sunder, Kesevan Dr. R. (2011), Computation of Reliability and Birnbaum Importance of Components of a Wind Turbine at High Uncertain Wind, International Journal of Computer Applications (0975 - 8887) Volume 32- No.4, October.

- Shaomin Wua, Frank P.A. Coolen (2013), A cost-based importance measure for system components: An extension of the Birnbaum importance, European Journal of Operational Research 225, 189-195.

- Tong, Y.L. (1985), A rearrangement inequality fort he longest run with an application to network reliability, Journal of Applied Probability, 22:386-393.

- Wang W., Loman J., Vassiliou P., (2004), Reliability Importance of Components in a Complex System, Reliability and Maintainability Annual Symposium - RAMS, IEEE Transactions on Reliability.

- Wu, S. (2005), Joint importance of multistate systems, Computers and Industrial Engineering 49(1), 63-75.

- Zhiqiang Cai, Shubin Si (2013), Hongyan Dui and Shudong Sun Relationship and Changing Analysis of Birnbaum Importance for Different Components with Bayesian Networks, Quality Technology\&Quantitative Management Vol. 10, No. 2, pp. 203219. 